\title{
RNAi: a novel strategy for the treatment of prion diseases
}

\author{
Qingzhong Kong
}

Department of Pathology, Case Western Reserve University, Cleveland, Ohio, USA.

\begin{abstract}
Prion disease refers to a group of fatal transmissible neurodegenerative diseases for which no pharmacological treatment is available. The cellular prion protein $\left(\mathrm{PrPC}^{\mathrm{C}}\right)$ is required for both prion replication and pathogenesis, and reducing $\operatorname{PrP}^{\mathrm{C}}$ levels has been shown to extend survival time after prion infection. RNA interference (RNAi) is a sequence-specific posttranscriptional gene silencing mechanism. In this issue of the JCI, Pfeifer et al. report that lentivector-mediated RNAi significantly reduced neuronal $\operatorname{PrP}^{C}$ expression; effectively suppressed accumulation of the infectious proteaseresistant form of $\operatorname{PrP}(\mathrm{PrPsc})$ in a persistently infected neuroblastoma cell line; and markedly slowed the progression of prion disease in a unique chimeric mouse model (see the related article beginning on page 3204). These findings indicate that lentivector-mediated RNAi could, in principle, be developed for the therapy of prion disease.
\end{abstract}

\section{Prion diseases}

Prion diseases, or transmissible spongiform encephalopathies, have 3 etiologies: genetic (due to pathogenic mutations of the gene encoding the prion protein $[\mathrm{PrP}])$, acquired (as a result of exogenous prion infection), and sporadic (of unknown causes) (1). Prion disease is caused by prions, in which a self-replicating, infectious proteaseresistant form of $\operatorname{PrP}$ (termed $\mathrm{PrPsc}^{\mathrm{Sc}}$ ) is the only essential component identified to date. $\mathrm{PrP}^{\mathrm{Sc}}$ multiplies through conversion of the normal cellular $\operatorname{Pr} P\left(\operatorname{PrP}^{\mathrm{C}}\right)$ (Figure 1) (1). Host $\operatorname{PrP}^{\mathrm{C}}$ is essential for prion propagation, since no prion replication occurs in mice devoid of $\operatorname{PrPC}^{\mathrm{C}}$ (2). Host $\mathrm{PrP}^{\mathrm{C}}$ expression is also essential for prion pathogenesis. When normal neural tissues were grafted to the brains of mice devoid of $\operatorname{Pr} \mathrm{PC}^{\mathrm{C}}$, only the grafted tissues developed neurodegeneration after prion infection, but the $\mathrm{PrP}^{\mathrm{C}}$-free brain tissues of the host were virtually unaffected despite the presence of large amounts of $\operatorname{PrPsc}^{\mathrm{Sc}}$ (3). These and other reports establish the central role of host $\mathrm{PrPC}^{\mathrm{C}}$ in both prion replication and prion pathogenesis.

Nonstandard abbreviations used: ALS, amyotrophic lateral sclerosis; dsRNA, double-stranded RNA; PPS, pentosan polysulphate; $\mathrm{PrP}$, prion protein; $\mathrm{PrPC}$, cellular $\mathrm{PrP} ; \mathrm{PrP}^{\mathrm{Sc}}$, infectious protease-resistant PrP; RNAi, RNA interference; shRNA, short hairpin RNA.

Conflict of interest: The author has declared that no conflict of interest exists.

Citation for this article: J. Clin. Invest. 116:3101-3103 (2006). doi:10.1172/JCI30663.

\section{Development of treatments for prion} disease: current status

Prion disease is invariably fatal, and no therapy is available. Because serious damage to the brain has already occurred before clinical symptoms manifest, prion disease treatments have aimed not to cure the disease but to slow disease progression (4). There have been significant efforts to develop treatments for prion disease with limited success since the 1960 s, as summarized in the recent reviews by Trevitt and Collinge (5) and Caughey et al. (6). Given the essential role of $\mathrm{PrP}$ in prion disease, 2 basic therapeutic strategies have been adopted: reduction of $\operatorname{PrPC}^{\mathrm{C}}$ levels and prevention of the conversion from $\mathrm{PrP}^{\mathrm{C}}$ to $\mathrm{PrP}^{\mathrm{S} c}$. Direct targeting of $\mathrm{PrP}^{\mathrm{PSc}}$ is not viable due to the lack of understanding about the mechanism of PrPsc clearance in vivo and the risk of exacerbating the disease (5). Reducing the levels of $\mathrm{PrPC}^{\mathrm{C}}$ expression is known to attenuate prion replication and prolong survival time, and reagents that interfere with the conversion process also suppress prion replication. A large number of methods/reagents have been tested in animal models, cultured cells, or cell-free in vitro conversion systems (refs. 5, 6, and references therein). These include hundreds of compounds, e.g., polycationic, polyanionic, polyphenolic, tetrapyrrolic, tetracyclic, tricyclic, and related compounds (including some antimalarials); phenothiazines, steroids, antihistamines, polyene antibiotics, RNA aptamers, dominant-negative proteins (certain PrP mutants, PrP-dimers), copper chelators and copper, inhibitors of signal transduction, $\beta$-sheet-breaker peptides, anti-PrP antibodies; as well as immunomodulation and reagents that cause temporary depletion of follicular dendritic cells. Some of these compounds, such as pentosan polysulphate (PPS), which has been in use for the treatment of osteoarthritis in dogs, appear to be promising (Figure 1). Extended intraventricular infusion of PPS has been shown to significantly increase survival time of prion-infected mice even when administered at the onset of clinical symptoms, but earlier treatment gave better results (7). Some other strategies also have potential (Figure 1). For example, a recent report showed that active DNA vaccination against $\mathrm{Pr}^{\mathrm{C}}$ dramatically delayed disease onset in a transgenic mouse model, but it was followed by rapid disease progression (8).

\section{RNAi}

RNA interference (RNAi) is a highly conserved, sequence-specific posttranscriptional gene-silencing mechanism, whereby siRNA targets homologous mRNA for degradation (9). siRNAs are generated from endogenous or exogenous double-stranded RNAs (dsRNAs) by the dsRNA endonuclease Dicer; subsequently, siRNA activates the RNA-induced silencing complex (RISC) to degrade the target mRNAs (9) (Figure 1). Rapid advancement in the understanding of RNAi in general, and mammalian RNAi in particular (10), makes it feasible to use RNAi to develop therapeutics for a variety of human diseases. The huge potential of RNAi in research and therapeutics has been reflected by the awarding of the $2006 \mathrm{Nobel}$ Prize in Medicine to Craig Mello of University of Massachusetts Medical School and Andrew Fire of Stanford University School of Medicine for their contributions to the discovery of RNAi.

There are major challenges in developing therapeutic approaches that utilize RNAi. Since RNAi requires expression of dsRNAs 
Other promising strategies

\begin{tabular}{|ll|}
\hline Compounds and chemicals & Reagents and vaccines \\
PPS (polyanionic) & Anti-PrP antibodies, PrP-dimers, dominant- \\
Porphyrins (cyclic tetrapyrroles) & negative PrP mutants, DNA vaccines \\
Amphotericin B and MS8209 (polyene antibiotics) & Oral vaccination with PrP-expressing \\
Copper & Salmonella typhimurium \\
& Phosphorothioate oligonucleotides \\
\hline
\end{tabular}

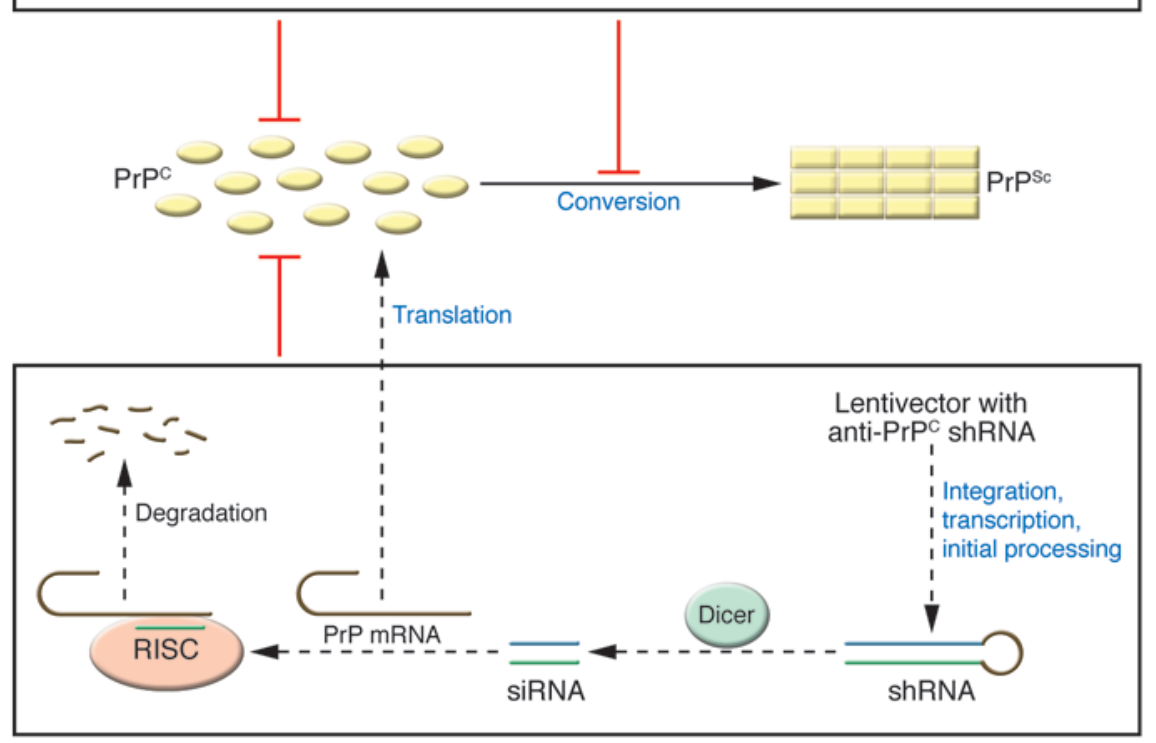

Lentivector-mediated RNAi

\section{Figure 1}

RNAi and other strategies for prion disease treatment. Monomeric PrPc (yellow ovals) converts

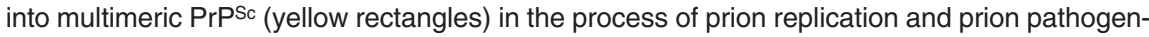
esis. Reagents or strategies that effectively reduce the PrPC level or interfere with the PrPC-toPrPsc conversion process have shown therapeutic potential for prion disease. In this issue of the $\mathrm{JCl}$, Pfeifer et al. (20) show that anti-PrPC shRNA carried on a lentivector is transfected into neuronal or ES cells, integrated into chromosomal DNA, and transcribed; anti-PrPC shRNA is released into the cytoplasm, where it is processed by endonuclease Dicer into siRNA. This in turn activates the RNA-induced silencing complex (RISC) to degrade PrP mRNAs, leading to reduced expression of $\mathrm{PrP}^{\mathrm{C}}$ and consequently diminished $\mathrm{PrPSc}$ accumulation and significantly improved survival time after prion infection. Other promising reagents and strategies are listed in the top box. Only the most effective and least toxic compounds or chemicals that have demonstrated therapeutic effects in animals after intracerebral prion inoculation are included; a few other reagents and vaccines that have potential but need further research are also listed (5-6).

including short hairpin RNAs (shRNAs), these dsRNAs could potentially activate the innate immune system, inhibit nontarget genes, or saturate the RNAi machinery (11-12). The biggest factor that hinders therapeutic application of RNAi is the need for a safe and efficient delivery system for dsRNAs. The lentiviruses are a family of retroviruses that can integrate into the genomes of not only dividing cells but also nondividing cells (such as neurons) to achieve stable, long-term expression of shRNAs. This feature makes lentivectors one of the favorite delivery systems for exogenous genes, especially in the central nervous system (13). Lentivector-mediated RNAi has been shown to be feasible for treatment of
Alzheimer disease and amyotrophic lateral sclerosis (ALS) in mouse models (14-15), and it is even being used for genome-scale loss-of-function screening (16). However, lentivectors have their own drawbacks: they require high-titer virus stocks; the consequences in the event that recipients are infected with related retroviruses are unknown; and their effectiveness may be reduced due to silencing of many integrated lentivector copies (17).

\section{Development of RNAi-based therapeutics for prion disease}

RNAi has demonstrable therapeutic potential in animal models of several neurodegenerative diseases, including Alzheimer disease
(14), ALS (15), spinocerebellar ataxia (18), and Huntington disease (19). The effectiveness of RNAi is particularly impressive in the ALS model, where it was reported to double the latency period and prolong the lifespan by approximately $80 \%$ after intramuscular injections of shRNAs (15).

In this issue of the JCI, Pfeifer et al. (20) report in vitro and in vivo data that raise hope for an RNAi-based therapy for prion disease. They showed that lentivector-mediated anti-PrPC ${ }^{\mathrm{C}}$ shNA expression effectively suppressed prion replication in a murine neuroblastoma cell line persistently infected with a mouse prion strain. More significantly, they also created chimeric mice using embryonic stem cells transfected with a lentivector carrying an anti-PrPC $\operatorname{shNA}$ and showed that the survival time after prion inoculation was markedly lengthened. In the chimeric mice, only cells derived from the lentivector-transfected embryonic stem cells expressed the anti-PrPC ${ }^{C}$ shNA, and the degree of chimerism was positively correlated with the increase in incubation time in general, implying that the degree of $\mathrm{PrP}^{\mathrm{C}}$ knock down is directly related to the improvement in survival time. These results indicate that lentivector-mediated expression of properly designed anti-PrPC shRNAs in neuronal cells has significant therapeutic effects in vitro and in vivo. It is also encouraging that no apparent abnormality was noted in the chimeric mice, suggesting that lentivector-mediated RNAi is well tolerated in at least the mouse model system. These data indicate that RNAi has therapeutic potential for prion disease, providing a novel venue for the search of an effective prion disease treatment. It may also be possible to utilize RNAi to prevent or delay the occurrence of prion disease in subjects carrying pathogenic mutations in the human PrP gene, PRNP.

Much more research is needed before RNAi can be harnessed to treat prion disease. Since Pfeifer et al. (20) demonstrated the therapeutic effect of the shRNAs only in chimeric mice where $\operatorname{Pr} P$ expression was suppressed from the embryonic stage in shRNA-expressing cells, future studies need to establish that shRNAs carried in a lentiviral vector can still prolong the lifespan of affected animals when administered into adult mice at least at presymptomatic stages of prion disease or, better yet, at early symptomatic stages of prion disease. It is noteworthy that only a moderate reduction in $\mathrm{PrP}^{\mathrm{C}}$ expression was observed in the brain area of adult mice where the lentivector was 
injected (see Figure 1, G and H, in ref. 20); even in the most highly chimeric mouse (approximately 95\% chimeric), the increase in survival time to 231 days was modest when compared with that of $167 \pm 1.2$ days for the control mice. These observations point to the need for a vast improvement of the lentivector to achieve more efficient transfection of neurons and much higher levels of shRNA expression in those cells that are transfected. A safe and effective protocol also needs to be developed to allow brain-wide lentivector transfection. Combined therapeutic strategies, such as application of both RNAi and PPS or other effective compounds (such as porphyrins), may hold real promise for the effective therapy of prion disease in the near future.

Address correspondence to: Qingzhong Kong, Department of Pathology, Case Western Reserve University, 2085 Adelbert Road, Cleveland, Ohio 44106, USA. Phone: (216) 368-1756; Fax: (216) 368-2546; E-mail: qxk2@case.edu.
1. Prusiner, S.B. 1998. Prions. Proc. Natl. Acad. Sci.U. S. A. 95:13363-13383.

2. Sailer, A., Bueler, H., Fischer, M., Aguzzi, A., and Weissmann, C. 1994. No propagation of prions in mice devoid of PrP. Cell. 77:967-968.

3. Brandner, S., et al. 1996. Normal host prion protein necessary for scrapie-induced neurotoxicity. Nature. 379:339-343.

4. Aguzzi, A., Glatzel, M., Montrasio, F., Prinz, M., and Heppner, F.L. 2001. Interventional strategies against prion diseases. Nat. Rev. Neurosici. 2:745-749.

5. Trevitt, C.R., and Collinge, J. 2006. A systematic review of prion therapeutics in experimental models. Brain. 129:2241-2265.

6. Caughey, B., et al. 2006. Prions and transmissible spongiform encephalopathy (TSE) chemotherapeutics: a common mechanism for anti-TSE compounds? Acc. Chem. Res. 39:646-653

7. Doh-Ura, K., et al. 2004. Treatment of transmissible spongiform encephalopathy by intraventricular drug infusion in animal models. J. Virol. 78:4999-5006

8. Fernandez-Borges, N., et al. 2006. DNA vaccination can break immunological tolerance to $\mathrm{PrP}$ in wild-type mice and attenuates prion disease after intracerebral challenge. J. Virol. 80:9970-9976.

9. Sen, G.L., and Blau, H.M. 2006. A brief history of RNAi: the silence of the genes. FASEB J. 20:1293-1299.

10. Collins, R.E., and Cheng, X. 2006. Structural and biochemical advances in mammalian RNAi. J. Cell. Biochem. 99:1251-1266.

11. Snove, O., Jr., and Rossi, J.J. 2006. Expressing short hairpin RNAs in vivo. Nat. Methods. 3:689-695.

12. Xia, X.G., Zhou, H., and Xu, Z. 2005. Promises and challenges in developing RNAi as a research tool and therapy for neurodegenerative diseases. Neurodegener. Dis. 2:220-231.

13. Li, C.X., et al. 2006. Delivery of RNA interference. Cell Cycle. 5:2103-2109.

14. Singer, O., et al. 2005. Targeting BACE1 with siRNAs ameliorates Alzheimer disease neuropathology in a transgenic model. Nat. Neurosci. 8:1343-1349.

15. Ralph, G.S., et al. 2005. Silencing mutant SOD1 using RNAi protects against neurodegeneration and extends survival in an ALS model. Nat. Med. 11:429-433.

16. Root, D.E., Hacohen, N., Hahn, W.C., Lander, E.S., and Sabatini, D.M. 2006. Genome-scale loss-offunction screening with a lentiviral RNAi library. Nat. Methods. 3:715-719.

17. Ellis, J. 2005. Silencing and variegation of gammaretrovirus and lentivirus vectors. Hum. Gene Ther. 16:1241-1246.

18. Xia, H., et al. 2004. RNAi suppresses polyglutamine-induced neurodegeneration in a model of spinocerebellar ataxia. Nat. Med. 10:816-820.

19. Harper, S.Q., et al. 2005. RNA interference improves motor and neuropathological abnormalities in a Huntington's disease mouse model. Proc. Natl. Acad. Sci. U. S. A. 102:5820-5825.

20. Pfeifer, A., et al. 2006. Lentivector-mediated RNAi efficiently suppresses prion protein and prolongs survival of scrapie-infected mice. J. Clin. Invest. 116:3204-3210. doi:10.1172/JCI29236.

\title{
Search for the preadipocyte progenitor cell
}

\author{
Gary J. Hausman ${ }^{1}$ and Dorothy B. Hausman ${ }^{2}$ \\ ${ }^{1}$ United States Department of Agriculture — Agricultural Research Service, Athens, Georgia, USA. ${ }^{2}$ University of Georgia, Athens, Georgia, USA.
}

\begin{abstract}
An increase in adipocyte number is a major contributor to the increase in adipose tissue mass that is characteristic of obesity. The identity and regulation of the adipocyte precursor cell (or preadipocyte) and the preadipocyte precursor cell (or progenitor cell) have been intensely studied for many years. In this issue of the JCI, Crossno et al. report that progenitor cells originating from outside the adipose tissue, in particular the bone marrow, can contribute to an increase in adipocyte number (see the related article beginning on page 3220). Their study in mice reveals that treatment with the thiazolidinedione rosiglitazone or exposure to a high-fat diet promotes the trafficking of circulating bone marrow-derived progenitor cells into adipose tissue, where they become multilocular adipocytes. This adds a new and unexpected dimension to this research arena.
\end{abstract}

\section{Historical perspective}

Early investigations noted that the full range of cells common to red bone marrow, including monocytes, reticular cells, lymphocytes, normoblasts, erythroblasts, and basophils, were observed in developing adi-

Nonstandard abbreviations used: TZD, thiazolidinedione.

Conflict of interest: The authors have declared that no conflict of interest exists.

Citation for this article: J. Clin. Invest. 116:3103-3106 (2006). doi:10.1172/JCI30666. pose tissue (1). In particular, a wide range of cell phenotypes common to red bone marrow, including endothelial cell precursors, pericytes, macrophages, immature macrophages, fibroblast-like perivascular reticular cells, and perivascular mesenchymal cells, were among those implicated as the adipocyte precursor cell (1). Adipose tissue and bone marrow-derived mesenchymal cells have a similar protein expression phenotype based on cell-surface markers $(2,3)$. Furthermore, adipogenic differentiation of both adipose tissue and bone marrow- derived mesenchymal cells are similarly characterized by increases in the expression of key adipocyte markers such as fatty acidbinding protein, lipoprotein lipase, PPAR $\gamma$, and CCAAT/enhancer-binding protein $\alpha$ $(\mathrm{C} / \mathrm{EBP} \alpha)(4,5)$. Bone marrow-derived adipocytes also secrete the adipocyte-specific factors leptin and adiponectin, indicating functional similarity between bone marrow-derived and adipose tissue-derived adipocytes (4). Interestingly, clusters of red bone marrow cell types have been observed in developing adipose tissue (6). The number of these cell types was associated temporally and spatially with the development of large clusters of multilocular (comprising many compartments) adipocytes representative of an immature stage of adipocyte development in white adipose tissue. These observations would seem to counter the long-held belief that new adipocytes arise solely from resident preadipocyte progenitors and hint that progenitor cells from tissues outside adipose depots could contribute to the formation of new adipocytes. In support of the latter concept, in this issue 